2010-01-01

\title{
Simple Design Technique for a Triangular FBG Filter Based on a Linearly Chirped Grating
}

\author{
Qiang Wu \\ Technological University Dublin, qiang.wu@tudublin.ie \\ Gerald Farrell \\ Technological University Dublin, gerald.farrell@tudublin.ie \\ Yuliya Semenova \\ Technological University Dublin, yuliya.semenova@tudublin.ie
}

Follow this and additional works at: https://arrow.tudublin.ie/engscheceart

Part of the Electrical and Computer Engineering Commons

\section{Recommended Citation}

Wu, Q.,Farrell, G. \&Semenova, Y. (2010) Simple Design Technique for a Triangular FBG Filter Based on a Linearly Chirped Grating. Optics Communications Volume 283, Issue 6, Pages 985-992. doi:10.1016/ j.optcom.2009.11.038

This Article is brought to you for free and open access by the School of Electrical and Electronic Engineering at ARROW@TU Dublin. It has been accepted for inclusion in Articles by an authorized administrator of ARROW@TU Dublin. For more information, please contact arrow.admin@tudublin.ie, aisling.coyne@tudublin.ie, gerard.connolly@tudublin.ie. 


\section{AUTHOR QUERY FORM}

\begin{tabular}{|l|l|l|}
\hline & Journal: OPTICS & Please e-mail or fax your responses and any corrections to: \\
& & E-mail: corrections.esnl@elsevier.sps.co.in \\
\hline ELSEVIER & Article Number: 14704 & Fax: +31204852799 \\
\hline
\end{tabular}

Dear Author,

Any queries or remarks that have arisen during the processing of your manuscript are listed below and highlighted by flags in the proof. Please check your proof carefully and mark all corrections at the appropriate place in the proof (e.g., by using on-screen annotation in the PDF file) or compile them in a separate list.

For correction or revision of any artwork, please consult http://www.elsevier.com/artworkinstructions.

Articles in Special Issues: Please ensure that the words 'this issue' are added (in the list and text) to any references to other articles in this Special Issue.

Uncited references: References that occur in the reference list but not in the text - please position each reference in the text or delete it from the list.

Missing references: References listed below were noted in the text but are missing from the reference list - please make the list complete or remove the references from the text.

\begin{tabular}{|l|l|}
\hline $\begin{array}{c}\text { Location in } \\
\text { article }\end{array}$ & Query / remark \\
\hline Please insert your reply or correction at the corresponding line in the proof
\end{tabular}

Electronic file usage

Sometimes we are unable to process the electronic file of your article and/or artwork. If this is the case, we have proceeded by: 
2 Simple design technique for a triangular FBG filter based on a linearly 3 chirped grating

4 Qiang $\mathrm{Wu}^{*}$, Gerald Farrell, Yuliya Semenova

5 Photonics Research Center, School of Electronic and Communications Engineering, Dublin Institute of Technology, Kevin Street, Dublin 8, Ireland

\begin{abstract}
A B S T R A C T
A novel and simple design technique for triangular spectrum response of fibre Bragg grating (FBG) is presented based on a linear chirped grating. It is shown that this method is fast and can give a straightforward solution to meet a design target. The numerical simulation examples verified the effectiveness of the design method. A general approach to design for multichannel triangular spectral responses for FBG filters is proposed, which provides a solution that achieves a minimum change of refractive index for the fibre.
\end{abstract}

(c) 2009 Elsevier B.V. All rights reserved.

\section{A R T I C L E I N F O}

\section{Article history:}

Received 26 September 2009

Received in revised form 6 November 2009

Accepted 14 November 2009

Available online $\mathrm{xxxx}$

\section{Keywords:}

Fibre Bragg grating

Edge filter

FBG sensor

Triangular filter

\section{Introduction}

ibre Bragg gratings (FBGs) has been widely used in optical communications and optical sensing because of their many advantages such as simplicity, low cost, high sensitivity, chemical resistance, multiplexing capabilities and immunity from electrical and magnetic interference [1]. In sensing applications, it was widely used in bridge [2], petroleum tube and coal mine safety monitoring, river surveillance monitoring, civil structural monitoring, aerospace health monitoring [3] etc. For all these applications, it is essential to interrogate the FBG sensor. Due to the high cost of an optical spectrum analyzer (OSA), it is necessary to develop a cost effective method to extract the wavelength information and thus interrogate the FBG sensor. Some FBG interrogation technologies have been developed such as ratiometric approach [4], unbalanced MachZehnder interferometers [5] and scanning Fabry-Perot filters [6] etc. Among these technologies, ratiometric wavelength measurement is a simple, high speed and cost effective scheme compared to wavelength scanning based active schemes [4-8]. In a ratiometric system, the characteristics of the edge filter such as slope and stability will significantly influence the resolution of the system. An FBG has a high stability and a large slope and thus is an ideal device for use as an edge filter and recently has been proposed for interrogation of a FBG sensor [9]. In [10] it was shown that a single-multiple-single mode fibre edge filter used to interrogate a FBG could also be used to compensate temperature induced errors

\footnotetext{
* Corresponding author.

E-mail address: qiang.wu@dit.ie (Q. Wu).
}

for the FBG sensor. Since a FBG based edge filter has the same wavelength shift vs. temperature characteristic as a FBG used for sensing, then a FBG edge filter can also compensate for temperature induced errors. Recently Littler [11] and Rochette [12] have developed an adjustable bandwidth FBG optical filter, which shows that the slope of a FBG edge filter could potentially be adjusted, to allow for an appropriate working wavelength range, by altering the bandwidth of the FBG edge filter.

As we know, the FBG normally has a flat top response for the reflective spectrum [13] which is not suitable for use as an edge filter. There are several papers concentrating on the design of a triangular reflective spectrum response of a FBG by using either a covariance matrix adapted evolution strategy algorithm [14] or an accelerated genetic algorithm [15]. These design methods are based on optimisation methods such as simulated annealing and a genetic algorithm which are complicated and time consuming. An inverse scattering method is a precise and computationally efficient method for FBG design [16]. However, this method still suffers from significant complexity. Longhi et al. proposed a simple method using first-order Born approximation [17] for FBG spectrum design. It works in the weak-grating condition (Born approximation), which assumes the reflective spectrum shape is proportional to the spatial refractive index modulation profile of the linear chirped FBG. When the refractive index modulation is strong $\left(>10^{-4}\right)$, this method fails. Recently Bandyopadhyay et al. reported an empirical design technique of linear edge filter by using an apodized linearly chirped fibre grating [18]. This technique is based on investigating the impact of different apodization functions on the spectrum response of a FBG which will not give a 
straightforward solution to the target. In this paper, a simple and fast design technique for a triangular FBG filter is proposed. By using this technique, a solution will be directly given by a non-linear function once a target spectrum is given. This greatly simplifies the design process and saves time and gives a straightforward solution to the desired spectrum. A general approach to design for multichannel triangular spectral responses for FBG filters is proposed, which allows the refractive index modulation to be minimized with a genetic algorithm.

\section{Theoretical analysis}

For a fibre Bragg grating, the effective refractive index modulation can be modeled as [19]

$\delta n_{\mathrm{eff}}(z)=\bar{\delta} n_{\mathrm{eff}}(z)\left\{1+v \cos \left[\frac{2 \pi}{\Lambda(z)} z\right]\right\}$

where $\bar{\delta} n_{\text {eff }}(z)$ is the "dc" index change spatially averaged over a grating period, $x$ is the fringe visibility of the index change and normally it is $1, \Lambda(z)$ is the grating period and can be expressed as

$\Lambda(z)=\Lambda_{0}\left(1+c_{\mathrm{p}} z\right)$

where $\Lambda_{0}$ is the nominal grating period and $c_{\mathrm{p}}$ is the linear chirp coefficient of the grating period. For a uniform grating, $c_{\mathrm{p}_{\wedge}}=0$ and its maximum reflectivity $R_{\max }$ and wavelength are [19]

$R_{\max }=\tanh ^{2}(\kappa L)$

$\lambda_{\max }=\left(1+\frac{\bar{\delta} n_{\mathrm{eff}}}{n_{\mathrm{eff}}}\right) \lambda_{\mathrm{B}}$

where $L$ is the grating length, $n_{\mathrm{eff}}$ is the fibre effective index, $\hat{\lambda}_{\mathrm{B}}=2 n_{\mathrm{eff}} \Lambda$ is the designed Bragg wavelength, $\kappa$ is "AC" coupling coefficient which can be expressed as [19]

$\kappa=\frac{\pi}{\lambda} \bar{\delta} n_{\text {eff }}$

A chirped grating of length $L$ can be divided into $N$ uniform gratings of length $L_{s}$ as illustrated in Fig. 1 .

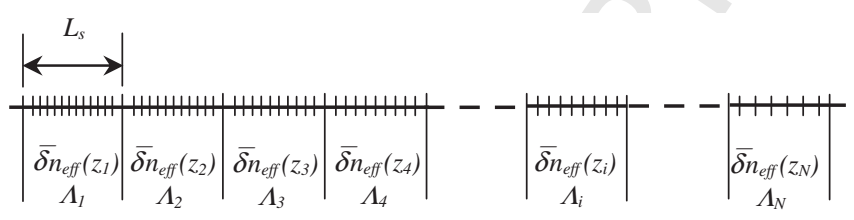

Fig. 1. Structure of ehirped fibre Bragg grating.

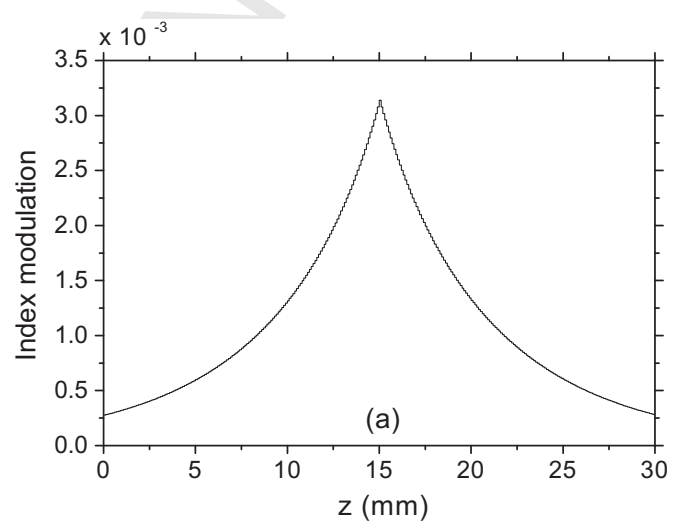

The chirped grating consists of $N$ uniform gratings. Each section has its own "dc" index change $\bar{\delta} n_{\text {eff }}(z)$ and grating period $\Lambda_{i}$. All the sections have the same length $L_{s}$ and

$\Lambda_{i}=\Lambda_{0}+c_{\mathrm{p}}\left(z_{i}-L / 2\right) \quad 0 \leqslant z_{i} \leqslant L$

Our design principle is to treat each section as a separate uniform grating with an individual centre wavelength and maximum reflectivity. In order to get a triangular spectrum response, we assume the maximum reflectivity of each section is a triangular function of wavelength, which can be defined either using a log scale

$\begin{cases}10 \lg \left\{\tanh ^{2}\left[\kappa(z) L_{s}\right]\right\}=a \lambda+b & \lambda_{0} \leqslant \lambda \leqslant \lambda_{1} \\ 10 \lg \left\{\tanh ^{2}\left[\kappa(z) L_{s}\right]\right\}=c \lambda+d \quad \lambda_{1} \leqslant \lambda \leqslant \lambda_{2}\end{cases}$

gr using a linear scale:

$\begin{cases}\tanh ^{2}\left[\kappa(z) L_{s}\right]=a \lambda+b & \lambda_{0} \leqslant \lambda \leqslant \lambda_{1} \\ \tanh ^{2}\left[\kappa(z) L_{s}\right]=c \lambda+d & \lambda_{1} \leqslant \lambda \leqslant \lambda_{2}\end{cases}$

where $a, b, c$ and $d$ are constants, and

$$
\begin{aligned}
\lambda_{0} & =2 n_{\mathrm{eff}}\left(\Lambda_{0}-c_{\mathrm{p}} L / 2\right) \\
\lambda_{1} & =2 n_{\mathrm{eff}}\left(\Lambda_{0}+c_{\mathrm{p}} z_{1}-c_{\mathrm{p}} L / 2\right) \\
\lambda_{2} & =2 n_{\mathrm{eff}}\left(\Lambda_{0}+c_{\mathrm{p}} L / 2\right) \\
\kappa(z) & =\frac{\pi}{\lambda_{\max }} \bar{\delta} n_{\mathrm{eff}}(z) \\
& =\frac{\pi}{2\left[n_{\mathrm{eff}}+\bar{\delta} n_{\mathrm{eff}}(z)\right]\left(\Lambda_{0}+c_{\mathrm{p}} z-c_{\mathrm{p}} L / 2\right)} \bar{\delta} n_{\mathrm{eff}}(z) .
\end{aligned}
$$

Once we set our desired triangle, the parameters $a, b, c$ and $d$ are given, then the refractive index change $\bar{\delta} n_{\text {eff }}(z)$ can be directly given by Eq. (7). By using the parameters of $\bar{\delta} n_{\text {eff }}(z)$ from (7), we can achieve a designed triangular spectrum response for the FBG.

\section{Numerical stimulations}

To demonstrate the effectiveness of the triangular spectrum design method above, numerical simulations on FBG were provided. The simulations were based on well-known transfer matrix method which was developed by Yamada et al. [20].

As a first pass, we assumed the target spectrum has a log scale with values $z_{1}=L / 2, \quad \lambda_{0}=1550 \mathrm{~nm}, \quad m=a \lambda_{0}+b=c \lambda_{2}+d=-25$, $n=a \lambda_{1}+b=c \lambda_{1}+\hat{d}=-5, \quad \widehat{c_{\mathrm{p}}}=1.57 \mathrm{~nm} / \mathrm{cm}, \quad$ and $L_{s}=100 \mu \mathrm{m}$, $L=30 \mathrm{~mm}$. By solving Eq. (7a) we can get the refractive index modûlation coefficient distribution $\bar{\delta} n_{\text {eff }}(z)$ along the grating length as shown in Fig. 2a. However, it was found that the value of $\bar{\delta} n_{\text {eff }}(z)$ we get from $(7 \hat{a})$ is so large that the reflectivity of the grating is

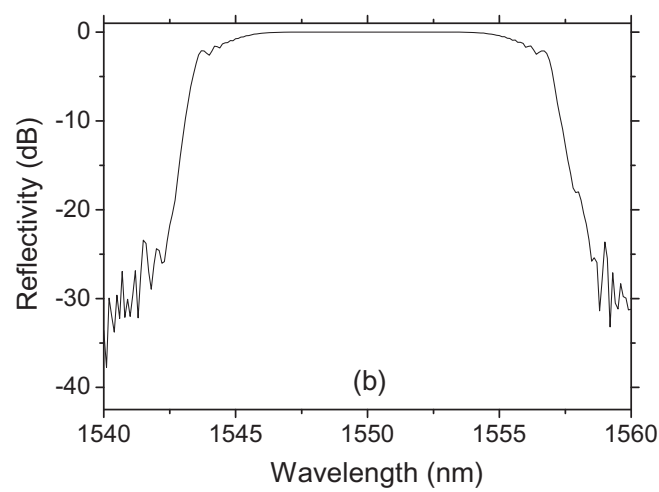

Fig. 2. Calculated (a) index modulation and (b) spectral response of the FBG. 
close to $100 \%$ resulting in a flat reflective spectrum response of the FBG which is shown in Fig. 2b.

To overcome this problem it is necessary to reduce the value of the refractive index modulation coefficient $\bar{\delta} n_{\text {eff }}(z)$ and keep its shape unchanged. Hence a scaling factor $W$ is applied to the calculated refractive index modulation coefficient $\bar{\delta} n_{\mathrm{eff}}(z) / W$ to get a desired triangular spectrum response. Fig. 3 shows the simulation results for the influence of scaling factor $W$ on the spectral response of the FBG.
Fig. 3 shows that with a reduced index modulation, a triangular reflective response for the FBG can be achieved when the peak reflectivity of the FBG is less than $90 \%$ (corresponding to $W>5$ in this case). As the scaling factor increases, the peak reflectivity decreases but the shape of the triangular spectral response remains unchanged.

In selecting a value for $W$, one approach is to assume a reasonable value of reflectivity, such as $50 \%$, a value of $W=10$ is therefore chosen for use in further simulations. Using a constant value of
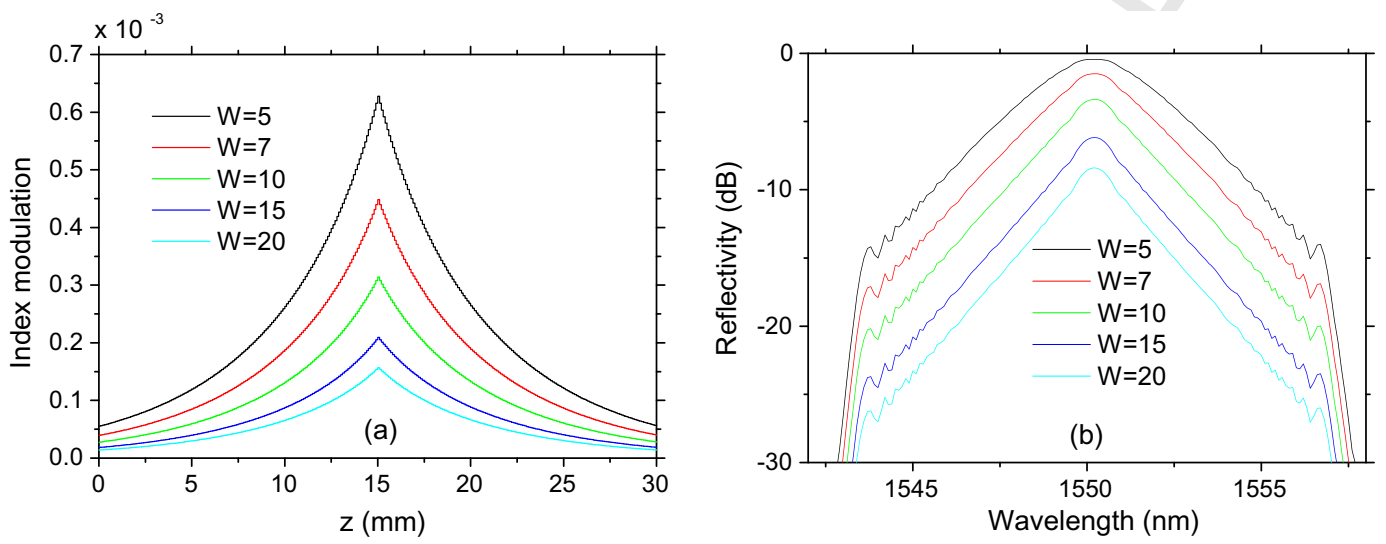

Fig. 3. Calculated (a) index modulation and (b) spectral response of the FBG by introducing a constant factor $W=5,7,10,15$ and 20 .
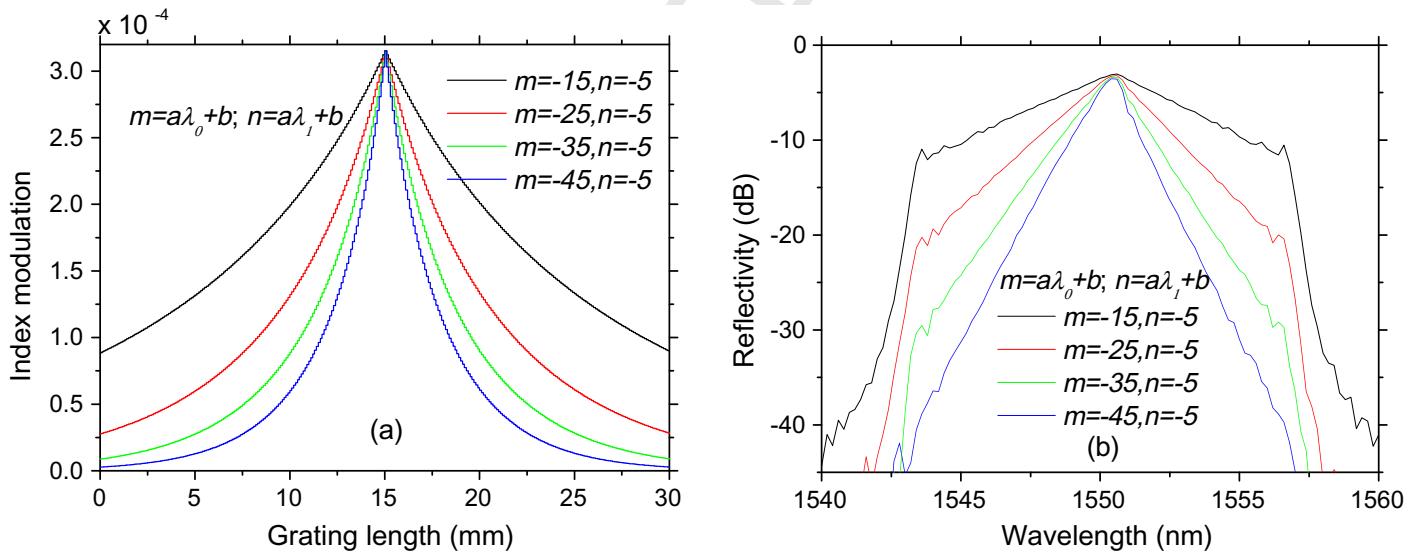

Fig. 4. Calculated (a) index modulation and (b) spectral response of the FBG with different shape.
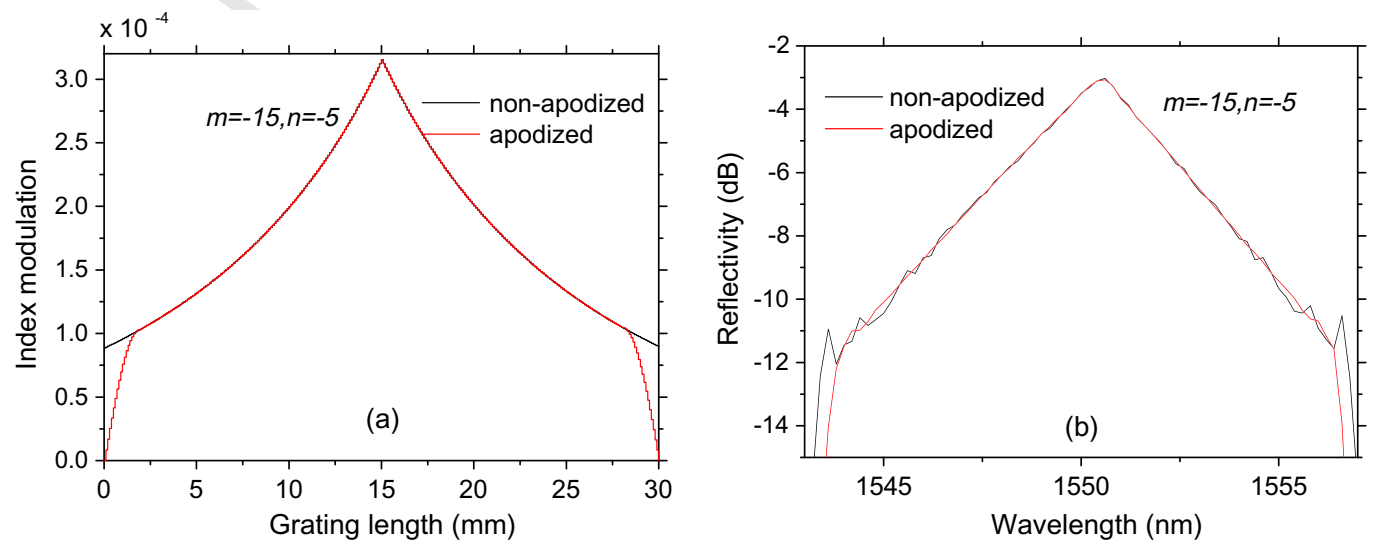

Fig. 5. Calculated (a) index modulation and (b) spectral response of the FBG without and with apodized index modulation. 
$W=10$ for the index modulation, an investigation on the effect of changes to $m$ and $n$ on the spectral response were carried out and are shown in Fig. 4.

By setting different values of $m$ and $n$, a triangular reflective response of FBG with different discrimination can be achieved simply by using the corresponding refractive index modulation shown in Fig. 4a. However, Fig. 4h also shows that the fluctuation of the reflectivity in the edge filter wavelength range is excessive especially for the FBG with $n=-15$ and $n=-5$. This is due to the non-apodized refractive index modulation at both sides of the grating. In order to eliminate the reflectivity fluctuation, an apodized refractive index modulation with a sin function is applied to both sides of the grating for a $2 \mathrm{~mm}$ length. Fig. 5 gives the simulated results for both non-apodized and apodized refractive index modulated FBG.

Fig. 5b shows that with an apodized index modulation as shown in Fig. 5a, the reflective spectrum of the FBG becomes much smoother. This indicates that apodization of the index modulation on both sides will reduce the fluctuation of the reflective spectrum of the designed FBG. All the simulations below are based on a sin function apodization for a $2 \mathrm{~mm}$ length for both sides of the grating.
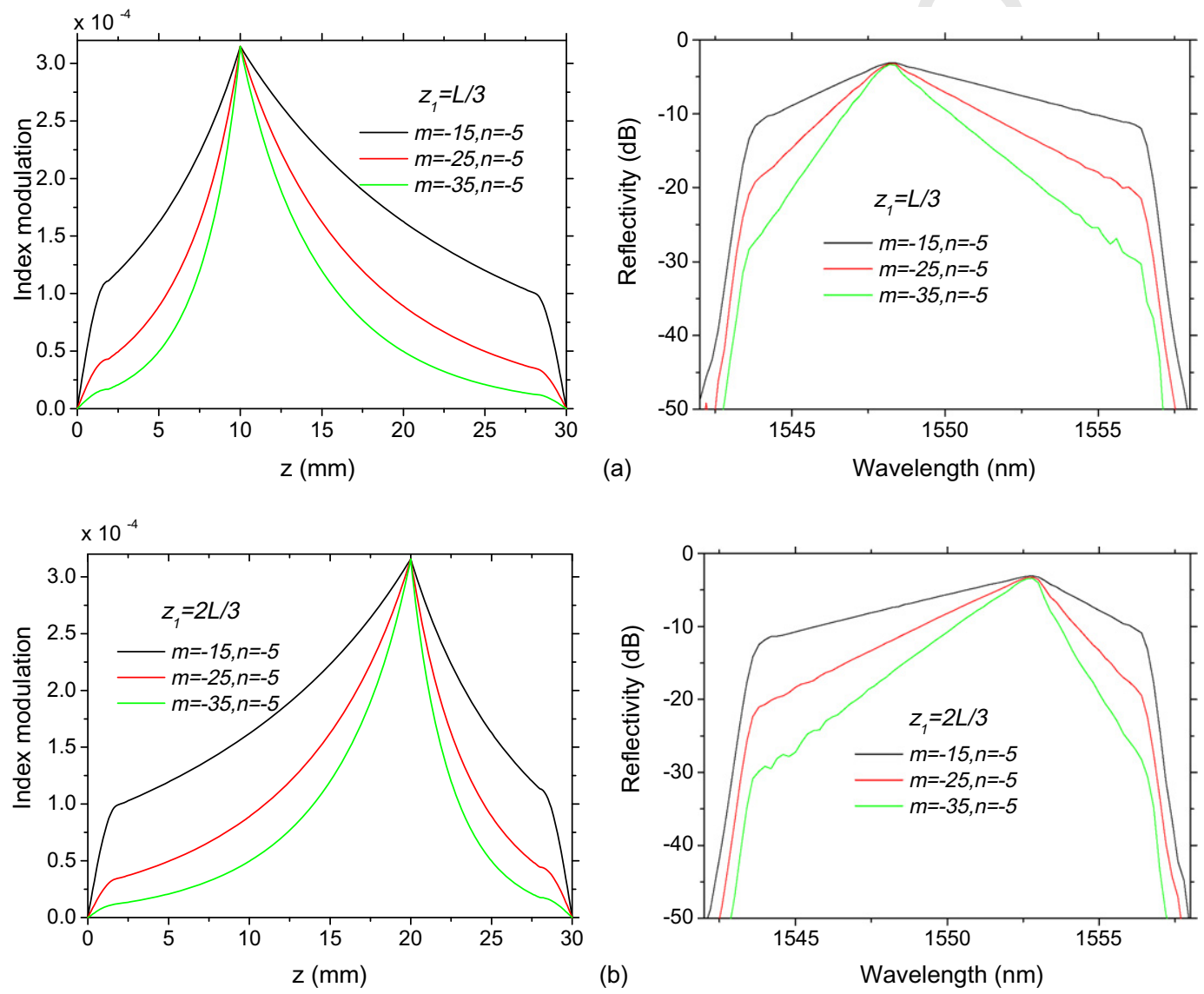

Fig. 6. Calculated index modulation and reflective spectral response of the FBG with (a) $z_{i}=L / 3$ and (b) $z_{i}=L / 1.5$.
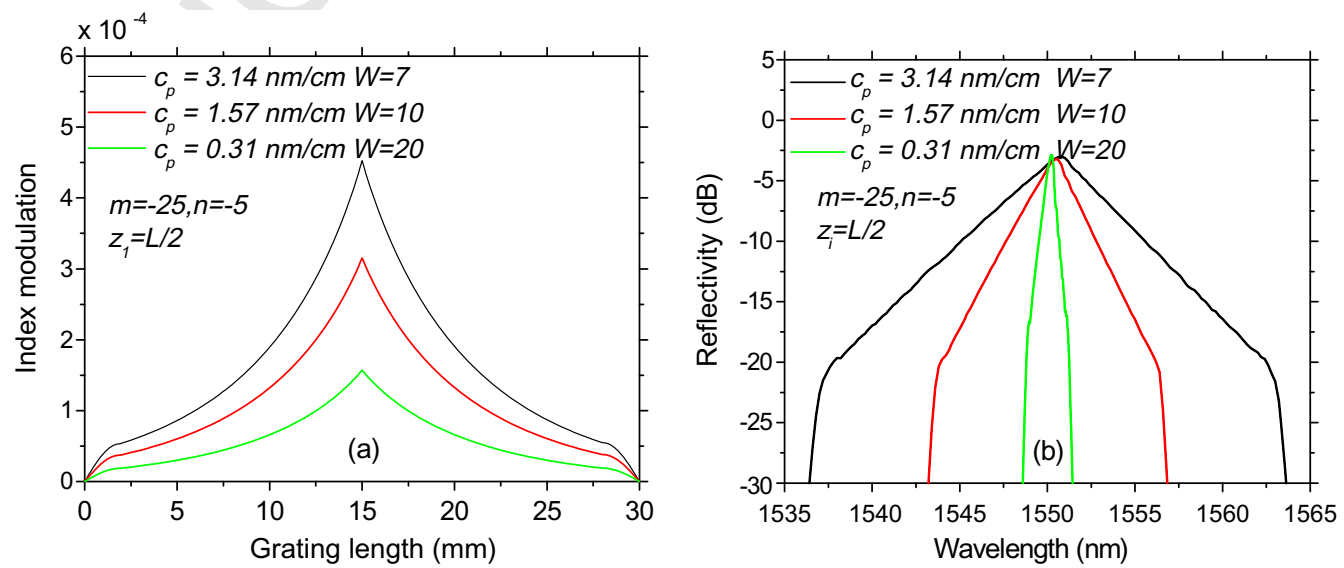

Fig. 7. Calculated (a) index modulation and (b) reflective spectral response of the FBG with $c_{\mathrm{p}}=3.14,1.57$ and $0.31 \mathrm{~nm} / \mathrm{cm}$, respectively. 
If we want to design an asymmetrical triangular spectrum, the only thing we need to do is to reset $z_{1}$ to a desired value and keeping other parameters unchanged. Fig. 6 gives simulation results for $z_{1}=L / 3,2 L / 3$, respectively.

Fig. 6 shows that by using the refractive index modulation provided, an asymmetrical triangular spectrum can be easily obtained.

To investigate the influence of linear grating period's chirp coefficient $c_{\mathrm{p}}$ on the reflective spectrum of the FBG, simulations were carried out by setting different $c_{\mathrm{p}}$. Fig. 7 gives the simulation results for $c_{\mathrm{p}}=3.14,1.57$ and $0.31 \mathrm{~nm} / \mathrm{cm}$, respectively.

Fig. 7 shows that with different $c_{\mathrm{p}}$, in order to have similar peak reflectivity, the FBG should have different refractive index modulation (and hence different scaling factor $W$ ). The larger the $c_{\mathrm{p}}$, the smaller the scaling factor $W$. In all cases, the designed gratings have triangular spectrum as shown in Fig. 7b. Fig. 7b also shows that the peak wavelength is slightly different for different $c_{\mathrm{p}}$. This is because comparing to a lower refractive index modulation, a
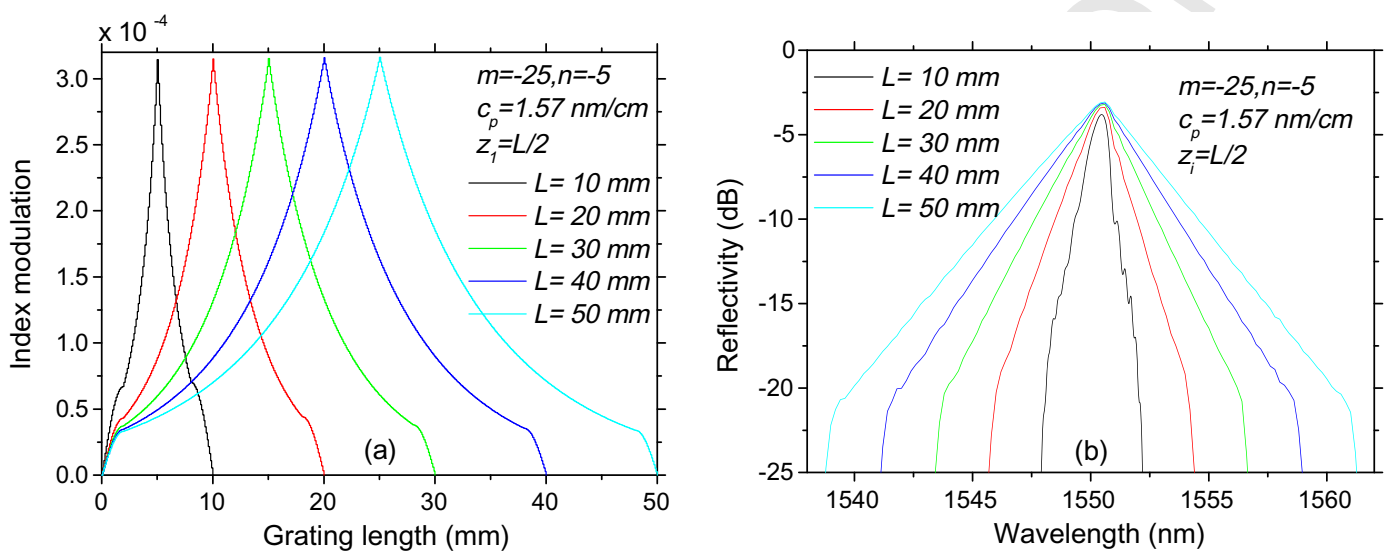

Fig. 8. Calculated (a) index modulation and (b) reflective spectrum response of the FBG with grating length $L=10,20,30,40$ and $50 \mathrm{~mm}$, respectively.
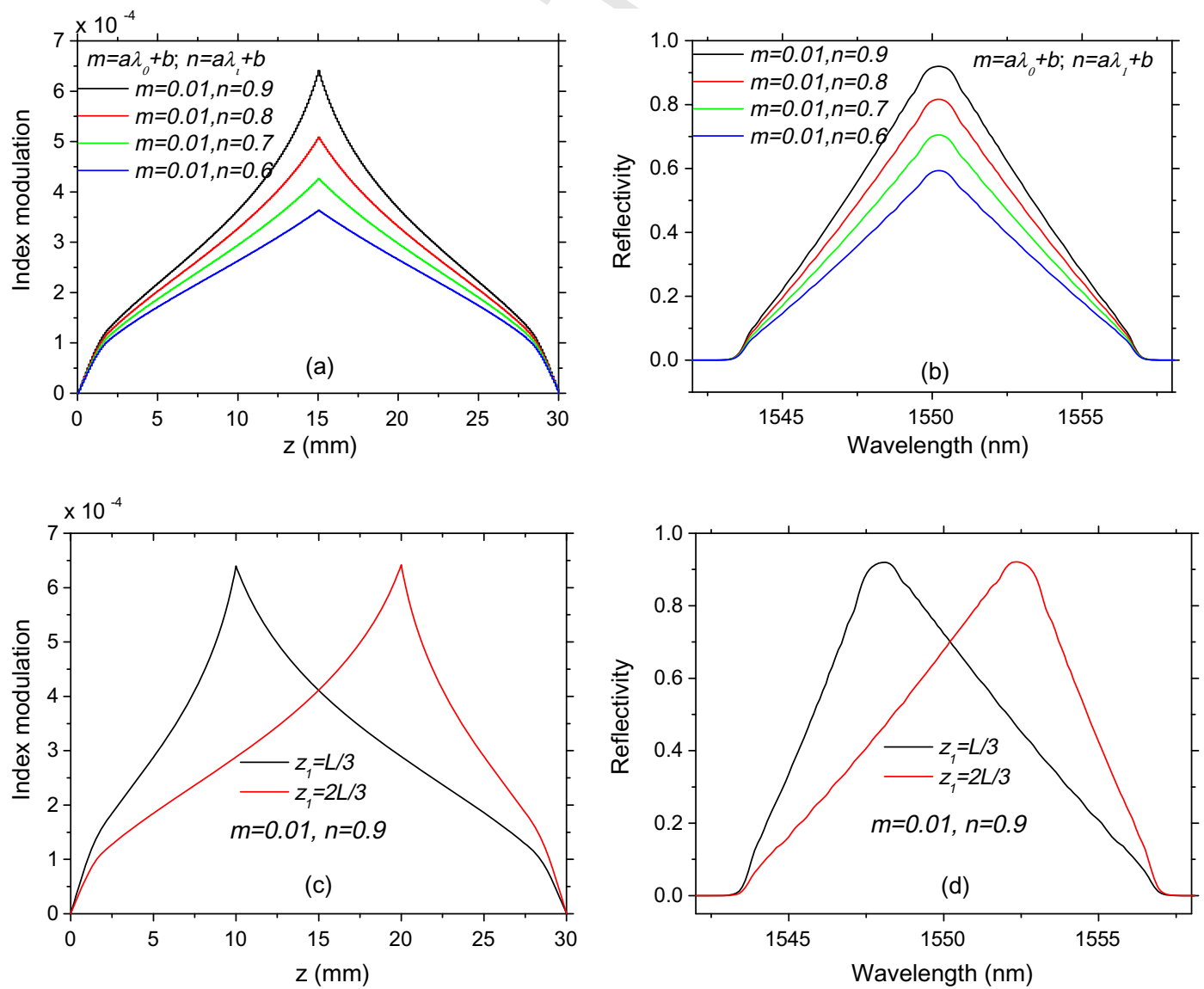

Fig. 9. Calculated (a) index modulation and (b) spectral response of the FBG with different reflectivity but a symmetrical triangular spectrum and (c) index modulation and (d) spectral response with an asymmetrical triangular spectrum. 
higher index modulation will result in a longer Bragg wavelength as described in Eq. $(9)_{2}$

The influence of grating length $L$ on the triangular spectrum response of designed FBG was also studied and the simulation results were shown in Fig. 8.

From Fig. 8 one can see that as grating length increases, the reflective spectrum response of the FBG becomes smoother at the triangular response area. This indicates that the design method in this paper is more suitable for a longer grating length.

Simulations for the target spectrum with a linear scale have been also carried out and the simulation results are shown in Fig. 9. In our simulation, the parameters used are: $z_{1}=L / 2$, $\lambda_{0}=1550 \mathrm{~nm}, \quad m=a \lambda_{0}{ }^{+} b=c \lambda_{2}{ }^{+} d, \quad n=a \lambda_{1}{ }^{+} b=c \lambda_{1}{ }^{+} d$, $c_{\mathrm{D}}=1.57 \mathrm{~nm} / \mathrm{cm}$, and $L_{S_{\wedge}}=100 \mu \mathrm{m}, \hat{K}=30 \mathrm{~mm}, W_{\hat{\lambda}}=14$. By solving Eq. (7b) we can get the refractive index modulation coefficient distribution $\bar{\delta} n_{\text {eff }}(z)$ along the grating length. In order to eliminate fluctuations in the reflectivity, an apodized refractive index modulation using a sin function is also applied to both sides of the grating for a $2 \mathrm{~mm}$ length.

Fig. 9 shows that with above design method, a triangular spectral response, using a linear scale, can be easily achieved.

The limitation in terms of the bandwidth is also investigated. To study this, a reflective bandwidth of $1 \mathrm{~nm}$ for a triangular spectrum was investigated but with two different sets of parameters: one grating has a long length $(120 \mathrm{~mm})$ but with a small chirp coefficient $(0.031 \mathrm{~nm} / \mathrm{cm})$; the other grating has a short length $(30 \mathrm{~mm})$ but with a relatively large chirp coefficient $(0.142 \mathrm{~nm} /$ $\mathrm{cm}$ ). Both gratings have a similar peak reflectivity of $90 \%$ and bandwidth of $1 \mathrm{~nm}$. The simulation results are shown in Fig. 10.
Fig. 10a shows that the shorter grating needs a higher refractive index modulation compared to longer grating in order to have a similar peak reflectivity. Fig. 10b shows that the longer length grating has smooth spectral response. This indicates that our method could be used to design a triangular spectrum with a bandwidth as low as $1 \mathrm{~nm}$. Our further investigations show that even when the bandwidth is as low as $0.5 \mathrm{~nm}$, the reflective spectral response is still smooth. However, as shown in Fig. 10b, the spectral response is not smooth when the grating length is less $30 \mathrm{~mm}$. This is a result of the trade-off between the filter bandwidth and grating length.

\section{Tolerance of the design method to fabrication errors}

The fabrication of a triangular FBG requires complex refractive index control and minor errors in fabrication will inevitably occur. We investigated the effect of a perturbation of the amplitude of the refractive index modulation on the designed FBG. To do this a perturbation factor $\alpha(z)$ is added to $\bar{\delta} n_{\text {eff }}(z)$ which can be expressed as follows:

$$
\delta^{\prime} n_{\mathrm{eff}}(z)={ }_{2} \delta n_{\mathrm{eff}}(z)[1+\alpha(z)]
$$

where $\bar{\delta} n_{\text {eff }}(z)$ is the required value obtained from Eq. (7). The typical distribution of fabrication errors follows a normal distribution. Thus we assume $\alpha(z)$ has a normal distribution with a mean 0 and standard deviation 1 (e.g. $|\alpha|<5 \%$ means that the value of $\alpha$ is generated randomly with a normal distribution with a mean 0 and deviation 1 in the range $\pm 5 \%$ ).
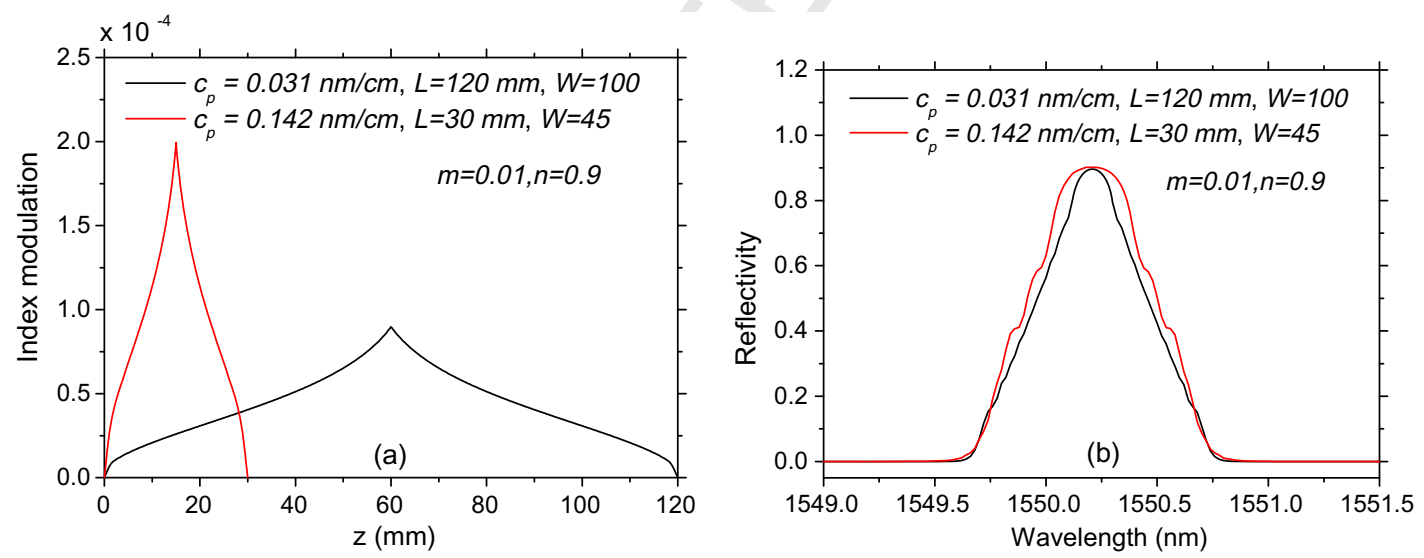

Fig. 10. Calculated (a) index modulation and (b) spectral response of the FBG with different grating length and chirp coefficient.
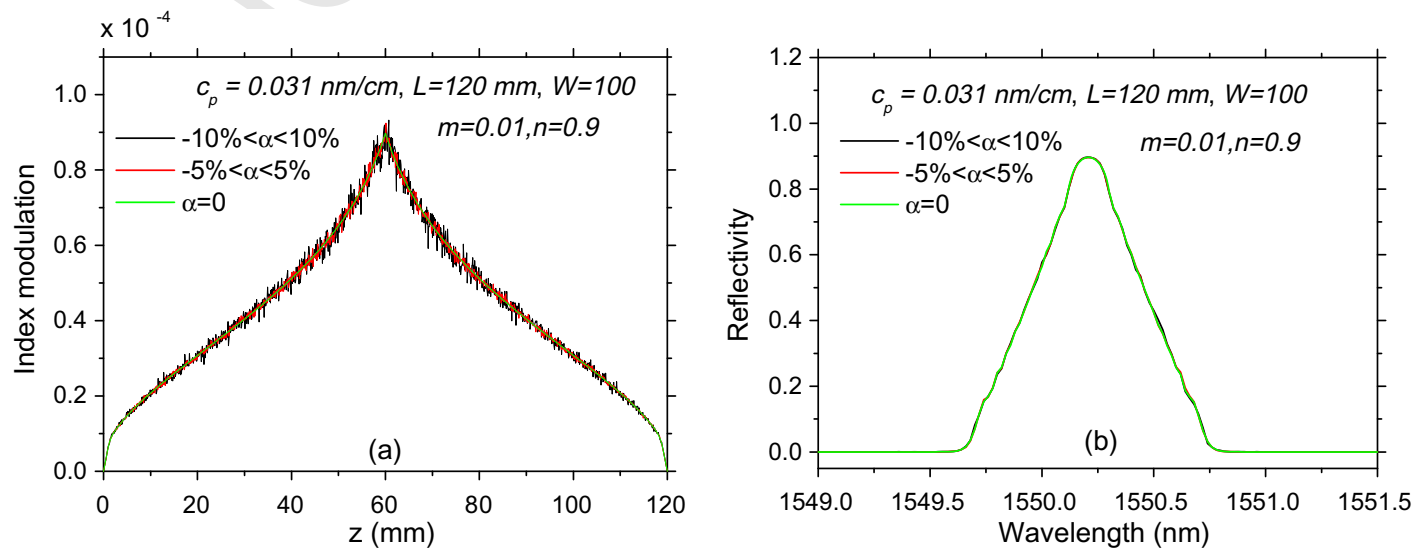

Fig. 11. Calculated (a) refractive index modulation perturbations and (b) the corresponding spectral response of the FBG. 

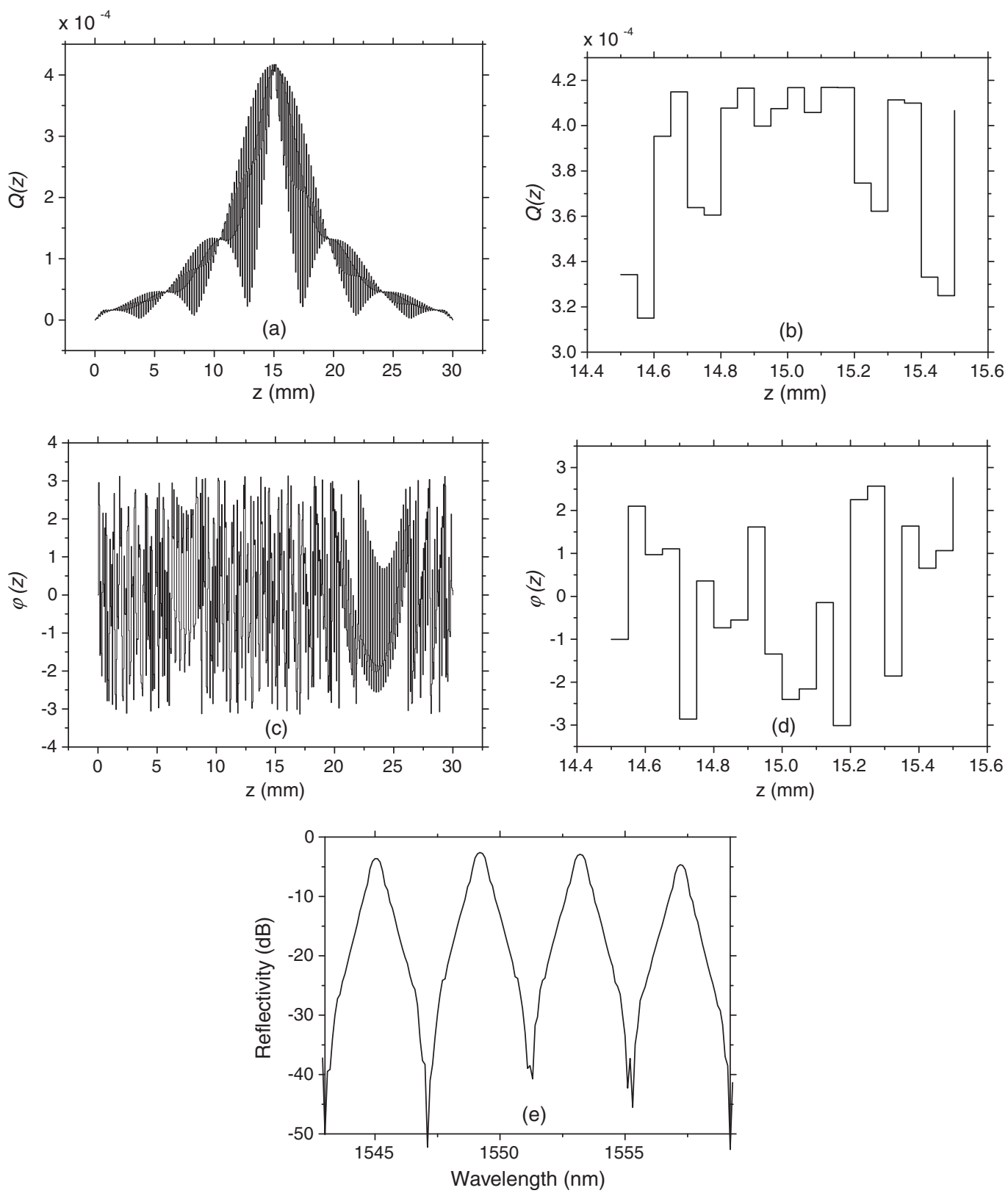

Fig. 12. Designed four-channel triangular FBG spectrum (a) amplitude profile (b) fine detail of the amplitude profile (c) phase profile (d) expanded section of the phase profile and (e) reflective spectrum.

Based on the method above, simulations were carried out using as an example the parameters for the longer grating in Fig. 10. The simulation results are shown in Fig. 11.

Fig. 11b shows a comparison of the reflective spectra when $|\alpha|=0,<5 \%$ or $<10 \%$. It can be seen that the corresponding variation of the reflectivity is small although the variations of the refractive index modulation are significant as shown in Fig. 11a. This indicates that when the refractive index modulation perturbation is in the range of $|\alpha|<10 \%$, the fabricated spectral response of a triangular FBG is still acceptable.

\section{Multichannel triangular spectra design}

It is also possible to use the design approach above to achieve multichannel triangular spectra by using the general multichannel design method in $[21,22]$. The general expression of $M$-channel
FBG takes a form of a superposition of $M$ individual constituent gratings:

$$
\begin{aligned}
Q(z) \exp \left\{i\left[K_{0} z+\varphi(z)\right]\right\}= & \sum_{m=1}^{M} \bar{\delta}_{m}(z) \exp \left[i \left(K_{0} z-K_{0} \Delta \lambda_{m} z / \lambda_{0}\right.\right. \\
& \left.\left.-K_{0} c_{\mathrm{p}} z^{2}+\phi_{m}\right)\right]
\end{aligned}
$$

where $Q(z)$ is the amplitude function of the composite grating and $K_{0}$ is its propagation constant and is related to the fundamental grating period $\Lambda_{0}$ by $K_{0}=2 \pi / \Lambda_{0} . \varphi(z)$ is the phase factor of the composite grating. $\bar{\delta} n_{\text {eff }}(z)$ and $\phi_{m}$ are the refractive index modulation and phase functions of the $m$ th constituent grating. $\Delta \lambda_{m}$ is the channel spacing of the $m$ th grating from the fundamental grating wavelength $\lambda_{0}$.

The complex Eq. (11) can be solved for the phase function $\phi(z)$ and the amplitude function $Q(z)$ to obtain 
$\varphi(z)=\tan ^{-1}\left[\frac{\sum_{m=1}^{M} \bar{\delta}_{m}(z) \sin \left[K_{0} z-K_{0} \Delta \lambda_{m} z / \lambda_{0}-K_{0} c_{\mathrm{p}} z^{2}+\phi_{m}\right]}{\sum_{m=1}^{M} \bar{\delta}_{m}(z) \cos \left[K_{0} z-K_{0} \Delta \lambda_{m} z / \lambda_{0}-K_{0} c_{\mathrm{p}} z^{2}+\phi_{m}\right]}\right]$

$Q(z)=\sqrt{\left(\sum_{m=1}^{M} \bar{\delta}_{m}(z) \cos \left[K_{0} z-K_{0} \Delta \lambda_{m} z / \lambda_{0}-K_{0} c_{\mathrm{p}} z^{2}+\phi_{m}\right]\right)^{2}+\left(\sum_{m=1}^{M} \bar{\delta}_{m}(z) \sin \left[K_{0} z-K_{0} \Delta \lambda_{m} z / \lambda_{0}-K_{0} c_{\mathrm{p}} z^{2}+\phi_{m}\right]\right)^{2}}$

Eqs. (12) and (13) are the design equations for multichannel triangular FBG filters. The amplitude refractive index modulation for a single FBG can be obtained from Eq. (7) and the complex phase and amplitude modulation can be solved from Eqs. (12) and (13). The amplitude function $\ell(z)$ can be minimized by systematically obtaining an optimal set of the phase $\phi_{m}$ of the constituent gratings as proposed by Kolossovski [23].

As an example, we simulated a four-channel triangular FBG filter. In our simulations, the parameters used are: $M=4$, $\lambda_{0}=1550 \mathrm{~nm}, n_{\mathrm{eff}}=1.485, z_{1}=L / 2, c_{\mathrm{p}_{\wedge}}=0.4 \mathrm{~nm} / \mathrm{cm}, \quad m=-35, \quad n=$ -5 , $L=30 \mathrm{~mm}$ and $\Delta \lambda=4 \mathrm{~nm}$.

As a result, the optimal set of $\phi_{m}$ is $\{2.074,3.808,1.117,5.984\}$ and the maximum amplitude modulation is $4.17 \times 10^{-4}$, which is only twice that of the single triangular FBG, that is $\hat{2} .09 \times 10^{-4}$. The calculated results were shown in Fig. 12a -e.

Fig. 12a-d shows the amplitude and phase modulation of the multichannel triangular FBG and Fig. 12e shows the reflective spectrum of the designed FBG. It can be seen that by using both amplitude and phase modulation with a grating length of only $30 \mathrm{~mm}$, we can obtain four channels with a triangular spectral response.

\section{Conclusion}

In this paper, we have presented a new and simple design method for a triangular spectral response for an FBG based on a linear chirped grating. This method can give a straightforward solution to the design target by only solving a non-linear function. Compared to the existed methods [14-16], this method is simple and fast. Moreover a multichannel design for a triangular spectrum is also provided based on our previous optimum design method and refractive index modulation can be minimized by using genetic algorithm. The numerical simulation examples verified that the proposed design method is effective.

\section{Acknowledgments}

This research was supported by Science Foundation Ireland.

\section{References}

[1] A.D. Kersey, M.A. Davis, H.J. Patrick, M. LeBlanc, K.P. Koo, C.G. Askins, M.A. Putnam, E.J. Friebele, J. Lightwave Technol. 15 (8) (1997) 1442.

[2] A. Kerrouche, J. Leighton, W.J.O. Boyle, Y.M. Gebremichael, T. Sun, K.T.V. Grattan, B. Taljsten, IEEE Sens. J. 8 (11-12) (2008) 2059.

[3] A. Hongo, S. Kojima, S. Komatsuzaki, Struct. Control Health Monit. 12 (3-4) (2005) 269.

[4] S.M. Melle, K. Liu, R.M. Measures, IEEE Photon. Technol. Lett. 4 (5) (1992) 516

[5] A.D. Kersey, T.A. Berkoff, IEE Electron. Lett. 28 (13) (1992) 1215.

[6] A.D. Kersey, T.A. Berkoff, W.W. Morsey, Opt. Lett. 18 (16) (1993) 1370.

[7] M.A. Davis, A.D. Kersey, Electron. Lett. 30 (1) (1994) 75.

[8] Y.P. Miao, B. Liu, W.H. Zhang, B. Dong, H.B. Zhou, Q.D. Zhao, IEEE Photon. Technol. Lett. 20 (13-16) (2008) 1393.

[9] R. Huang, Y.W. Zhou, H.W. Cai, R.H. Qu, Z.J. Fang, Opt. Commun. 229 (2004) 197.

[10] Q. Wu, A.M. Hatta, Y. Semenova, G. Farrell, Appl. Opt. 48 (2009) 5451.

[11] I.C.M. Littler, M. Rochette, B.J. Eggleton, Opt. Express 13 (2005) 3397.

[12] M. Rochette, I.C.M. Littler, R.W. McKerracher, B.J. Eggleton, IEEE Photon. Technol. Lett. 17 (8) (2005) 1680.

[13] K.O. Hill, Y. Fujii, D.C. Johnson, B.S. Kawasaki, Appl. Phys. Lett. 32 (10) (1978) 647.

[14] S. Baskar, P.N. Suganthan, Q. Ngo, A. Alphones, R.T. Zheng, Opt. Commun. 260 (2006) 716.

[15] J.C.C. Carvalho, M.J. Sousa, C.S.S. Junior, J.C.W.A. Costa, C.R.L. Frances, M.E.V. Segatto, Opt. Express 14 (22) (2006) 10715.

[16] J. Skaar, L. Wang, T. Erdogan, IEEE J. Quant. Electron. 37 (2) (2001) 165.

[17] S. Longhi, M. Marano, P. Laporta, V. Pruneri, IEEE Photon. Technol. Lett. 12 (11) (2000) 1498.

[18] S. Bandyopadhyay, P. Biswas, A. Pal, S.K. Bhadra, K. Dasgupta, J. Lightwave Technol. 26 (24) (2008) 3853.

[19] T. Erdogan, J. Lightwave Technol. 15 (8) (1997) 1277.

[20] M. Yamada, D. Sakuda, Appl. Opt. 26 (16) (1987) 3473.

[21] Q. Wu, P.L. Chu, H.P. Chan, J. Lightwave Technol. 24 (3) (2006) 1571.

[22] Q. Wu, C. Yu, K. Wang, X. Wang, Z. Yu, H.P. Chan, IEEE Photon. Technol. Lett. 17 (2) (2005) 381.

[23] K. Kolossovski, R. Sammut, A. Buryak, D. Stepanov, Opt. Express 11 (9) (2003) 1029. 